LAWRENCE LIVERMORE NATIONAL LABORATORY

Including the Effects of

Temperature-Dependent Opacities in the Implicit Monte Carlo Algorithm

N. A. Gentile

February 11, 2011

Journal of Computational Physics 
This document was prepared as an account of work sponsored by an agency of the United States government. Neither the United States government nor Lawrence Livermore National Security, LLC, nor any of their employees makes any warranty, expressed or implied, or assumes any legal liability or responsibility for the accuracy, completeness, or usefulness of any information, apparatus, product, or process disclosed, or represents that its use would not infringe privately owned rights. Reference herein to any specific commercial product, process, or service by trade name, trademark, manufacturer, or otherwise does not necessarily constitute or imply its endorsement, recommendation, or favoring by the United States government or Lawrence Livermore National Security, LLC. The views and opinions of authors expressed herein do not necessarily state or reflect those of the United States government or Lawrence Livermore National Security, LLC, and shall not be used for advertising or product endorsement purposes. 


\title{
Including The Effects Of Temperature-Dependent Opacities In The Implicit Monte Carlo Algorithm
}

\author{
N. A. Gentile ${ }^{1}$ \\ Lawrence Livermore National Laboratory, L-38, P. O. Box 808, Livermore California 94550
}

\begin{abstract}
The Implicit Monte Carlo technique of Fleck and Cummings [1] is often employed to numerically simulate radiative transfer. This method achieves greater stability than one with a fully explicit time discretization by estimating the $t^{n+1}$ value of $T^{4}$ from the thermal emission term, which is proportional to $T^{4}$. In the Fleck and Cummings algorithm, this results in decreasing the absorption by the so-called "Fleck factor", and adding a corresponding amount of effective scattering. We show how to include the effects of the temperature-dependent opacity to the estimated $t^{n+1}$ value of the thermal emission term. This results in the addition to the "Fleck factor" of a term that depends on $\frac{\mathrm{d} \sigma}{\mathrm{d} T}$. We demonstrate that this modification allows for more accurate solutions with much larger time steps for problems with opacities that have a strong temperature dependence.
\end{abstract}

\section{Introduction}

The time-dependent transport equation for photons in the absence of scattering and external sources is [2]

$$
\frac{1}{c} \frac{\partial I(\Omega, v)}{\partial t}+\Omega \cdot \nabla I(\Omega, v)=-\sigma(v, T) I(\Omega, v)+\sigma(v, T) B(v, T)
$$

where $c$ is the speed of light, $\sigma$ is the macroscopic absorption cross section in inverse length units. $B(v, T)$ is the Planck function

$$
B(v, T)=\frac{2 h v^{3}}{c^{2}} \frac{1}{\exp \left(\frac{h v}{k T}\right)-1}
$$

where $h$ is Planck's constant and $k$ is Boltzmann's constant. (We are using exp to symbolize the exponential function to avoid confusion with the symbols $e_{m}$ and $e_{r}$, which are used in this work to represent matter and radiation energy density.)

The thermal emission term, $\sigma B$, can be written

$$
\sigma(v, T) B(v, T)=\frac{1}{4 \pi} \frac{\sigma(v, T) b(v, T)}{\sigma_{P}(T)} \sigma_{P} a c T^{4}
$$

${ }^{*}$ Corresponding author. Tel + 19254238318 ; fax + 925423 9208; e-mail: gentile1@1lnl.gov Preprint submitted to Elsevier 
where $a$ is the radiation constant, $T$ is the matter temperature, $b(v, T)$ is the Planck distribution normalized over frequency, defined by

$$
b(v, T) \equiv \frac{15}{4 \pi} \frac{\left(\frac{h v}{k T}\right)^{3} \frac{h}{k T}}{\exp \left(\frac{h v}{k T}\right)-1},
$$

and $\sigma_{P}$ is the Planck mean opacity

$$
\sigma_{P}(T)=\int_{0}^{\infty} \sigma(v, T) b(v, T) \mathrm{d} v
$$

The expression $\frac{\sigma(v, T) b(v, T)}{\sigma_{P}(T)}$ is the probability distribution function for the frequency of the thermally emitted photons.

The transport equation is coupled to the material energy balance equation [2]

$$
\frac{\partial e_{m}}{\partial t}=\rho c_{v} \frac{\partial T}{\partial t}=\int_{4 \pi} \int_{0}^{\infty} \sigma(v, T) I(v, \Omega) \mathrm{d} \Omega \mathrm{d} v-\int_{4 \pi} \int_{0}^{\infty} \sigma(v, T) B(v) \mathrm{d} \Omega \mathrm{d} v .
$$

Here, $e_{m}$ is the matter energy density in units of energy per volume, $\rho$ is the mass density, and $c_{v}$ is the specific heat capacity in units of energy per mass per temperature. (Henceforth, to simplify notation, double integrals over the entire range of $\Omega$ and $v$ will be denoted with unadorned integrals. That is, we will write

$$
\int_{4 \pi} \int_{0}^{\infty} f(\Omega, v) \mathrm{d} \Omega \mathrm{d} v
$$

as

$$
\int f(\Omega, v) \mathrm{d} \Omega \mathrm{d} v
$$

where it is not confusing to do so.)

These equations can be solved by a Monte Carlo method described in [3]. The method discretizes the problem on a mesh. Each zone has a temperature and an absorption cross section. Particles representing photons are created in the zones at the beginning of each time step according to the emission term in the transport equation. Then the photons are followed through the zones, heating them according to the absorption term in Eq. (1). The zone temperatures are updated at the end of the time step, using Eq. (6), and the process is repeated.

This method becomes unstable when time steps of the order of

$$
\Delta t=\frac{\rho c_{v}}{a T^{3} c \sigma_{P}}
$$

are taken [1]. This instability occurs when the matter and radiation fields exchange an amount of energy comparable to the amount of energy necessary to change the matter temperature a nonnegligible amount in one time step. If the matter is only able to absorb energy during a time step, but is not able to re-radiate, as in the algorithm in [3], then instabilities may occur. The inability of the matter to re-radiate the energy it absorbs from the radiation during a time step is caused by the fact that the temperature in the emission term of the transport equation is calculated using the temperature at the beginning of the time step.

A method for solving the photon transport equation with improved stability when large time steps are taken was provided by Fleck and Cummings [1]. The method was dubbed Implicit Monte Carlo, usually abbreviated IMC. IMC works by using the matter energy balance equation 
to estimate the $t^{n+1}$ value of $T^{4}$ in the thermal emission term, Eq. (3). This estimate is substituted into the thermal source term in the transport equation. This substitution has the effect of reducing the absorption opacity in the transport equation by a factor of

$$
f=\frac{1}{1+\beta c \Delta t \sigma_{P}}
$$

and adding an equal amount of thermally redistributed isotropic scattering. Here $\beta \equiv 4 a T^{3} / \rho c_{v}$. This change allows the calculation to be run with much larger time steps before instabilities arise [4]. The quantity $f$ defined by Eq. (10) is sometimes referred to as the "Fleck factor".

The factor $f$ is small when photons are being absorbed and quickly re-emitted by the matter. Problems in which this occurs are said to exhibit tight coupling between the radiation and matter. IMC replaces the absorption and rapid reemission occurring in tightly coupled problems with isotropic scattering. This scattering is usually referred to as the effective scattering, to distinguish it from physical scattering. The effective scattering cross section $\sigma_{s}=(1-f) \sigma_{P}$.

The enhanced stability of IMC makes it a practical method for radiation transport simulations. It is often used in coupled radiation-hydrodynamics simulations, especially high energy density applications like inertial confinement fusion simulations. An overview of the use of IMC in high energy density simulations is provided in [5].

If the opacity is not a strong function of temperature, then using a $t^{n+1}$ estimate of $T^{4}$ with the $t^{n}$ value of $\sigma$ is a good estimate of the $t^{n+1}$ value of $\sigma B$. It will be a poor estimate when the temperature derivative of the opacity is a significant fraction of the temperature derivative for $\sigma B$. Since

$$
\frac{\mathrm{d} \sigma B}{\mathrm{~d} T}=\frac{\mathrm{d} \sigma}{\mathrm{d} T} a T^{4} b+\sigma \frac{\mathrm{d}\left(a T^{4} b\right)}{\mathrm{d} T},
$$

we expect the estimate used in Fleck and Cummings IMC to be inaccurate when the second term in Eq. (11) is large compared to the first. This will be true when

$$
\frac{T}{\sigma}\left|\frac{\mathrm{d} \sigma}{\mathrm{d} T}\right| \geq 4
$$

When Eq. (12) holds, IMC may produce inaccurate results. A typical failure mode (which will be demonstrated below) will occur when an initially cold material, with $\sigma \sim T^{-5}$ is subjected to a thermal radiation source with a radiation temperature $T_{r}$ large enough to change the temperature significantly. Since IMC will use the large $t^{n}$ value of the opacity throughout the time step, the matter temperature can increase to a value higher than the source radiation temperature in the first time step. This is unphysical, because thermodynamic consistency requires $T \leq T_{r}$. In subsequent time steps, the opacity will be small, because the matter temperature is very large. This reduces the value of $\sigma B$ in later time steps, so the matter does not radiate, and the unphysically large temperature persists.

The following sections will describe this problem with IMC further, and develop a modification of the IMC algorithm that reduces it for some simulations. In section 2, we describe a semi-implicit discretization of the source terms in the transport equation and the matter energy equation, including the temperature dependence of the opacity, and show that it results in a modification of the Fleck and Cummings algorithm. This modification introduces two new features. The first is a new term in the "Fleck factor" that depends on the derivative of the opacity with respect to temperature. The second is frequency and angle dependence added to the effective scattering and the thermal emission term. In section 3, we develop an approximation to the new 
effective scattering that makes its use in Monte Carlo simulations more tractable. In section 4, we apply this modified IMC method to two test problems with temperature dependent opacity. We show that it can produce more accurate answers with much larger time steps than Fleck and Cummings IMC on problems where the opacity has a strong temperature dependence.

\section{Semi-Implicit Discretization Of The Transport And Matter Energy Equations}

The system of Eqs. (1) and (6) is well-suited for solution by a Monte Carlo technique because it is linear in $I$. The equations are not linear in $T$, however. This could be dealt with by using some fixed values over each time step for $T, \sigma$, etc. on the right-hand side. The most obvious values to use are the $t^{n}$ values. However, as mentioned above, [3] and [1] showed that this method is unstable for large $\sigma$ at relatively small values of $\Delta t$. Equations which exhibit this behavior are said to be stiff. A semi-implicit treatment of the source terms in stiff equations can often allow numerical solutions with larger time steps (see, for example, [6]). Following an idea first presented in [7], we will apply a semi-implicit discretization to the source terms of Eqs. (1) and (6).

The semi-implicit discretization is a first order discretization that approximates the $t^{n+1}$ values of the sources by $t^{n}$ values plus derivatives of the source with respect to the independent variables [6]. For example, a semi-implicit discretization of a system of non-linear differential equations for $y_{i}$

would be

$$
\frac{\mathrm{d} y_{i}}{\mathrm{~d} t}=F_{i}(y)
$$

$$
\frac{y_{i}^{n+1}-y_{i}^{n}}{\Delta t}=F_{i}\left(y_{i}^{n}\right)+\sum_{j} \frac{\partial F_{i}}{\partial y_{j}}\left(y_{j}^{n+1}-y_{j}^{n}\right),
$$

where the derivatives of $F_{i}$ with respect to $y_{j}$ are evaluated at $t^{n}$. We have also approximated the time derivative of $y$ with a finite difference.

Applying the semi-implicit discretization to the system of equations results in a linear equation for $y_{i}^{n+1}$. The source term now depends on $t^{n+1}$ values of $y_{i}$, albeit using a linear approximation involving derivatives at $t^{n}$. This linear dependence usually makes numerical solutions of Eq. (14) more stable than explicit numerical solutions of Eq. (13), which use $t^{n}$ values for the source $F_{i}(y)$.

We would like to take advantage of the increased stability that the semi-implicit method provides for the numerical solution of Eqs. (1) and (6). However, since we intend to solve Eq. (1) by a Monte Carlo method, we will not discretize the time derivative of the radiation intensity to produce a linear equation for $I^{n+1}$. This is because we can solve the linear differential equation for $I$ which results from the semi-implicit approximation by a Monte Carlo method. So our semiimplicit approximation will involve expanding the source terms in Eqs. (1) and (6) in terms of $T^{n}$ and derivatives with respect to $T$ evaluated at $t^{n}$, and keeping terms up to first order in the differences.

For Eq. (1), this procedure results in

$$
\begin{aligned}
\frac{1}{c} \frac{\partial I}{\partial t}+\Omega \cdot \nabla I & =-\left(\sigma^{n}+\frac{\partial \sigma^{n}}{\partial T} \Delta T\right) I \\
& +\left(\sigma^{n}+\frac{\partial \sigma^{n}}{\partial T} \Delta T\right)\left(B^{n}+\frac{\partial B^{n}}{\partial T} \Delta T\right)
\end{aligned}
$$


where $\Delta T=T^{n+1}-T^{n}$. Eq. (15) is to be regarded as a differential equation to be solved over $\left[t^{n}, t^{n}+\Delta t\right]$.

Applying the procedure to Eq. (6) results in

$$
\begin{aligned}
\frac{\Delta e_{m}}{\Delta t} & =\rho c_{v}^{n} \frac{\Delta T}{\Delta t} \\
& =\int\left(\sigma^{n}+\frac{\partial \sigma^{n}}{\partial T} \Delta T\right) I \mathrm{~d} \Omega \mathrm{d} v \\
& -\int\left(\sigma^{n}+\frac{\partial \sigma^{n}}{\partial T} \Delta T\right)\left(B^{n}+\frac{\partial B^{n}}{\partial T} \Delta T\right) \mathrm{d} \Omega \mathrm{d} v .
\end{aligned}
$$

In Eq. (16), we have used the $t^{n}$ value of the heat capacity, $c_{v}^{n}$. This does not require us to use $c_{v}^{n}$ to obtain the $t^{n+1}$ value of the matter energy density. That can be obtained by inverting the equation of state once the emission and absorption have been calculated. The $t^{n}$ value of the heat capacity is used only in calculating the $t^{n}$ derivatives used in the semi-implicit approximation.

Henceforth, where $I$ multiplies a derivative with respect to $T$, we will use the $t^{n}$ value. We will also use a prime to denote differentiation with respect to $T$ evaluated at time $n$. For example, $B^{\prime} \equiv \frac{\partial B^{n}}{\partial T}$. This is done to simplify the notation.

In comparison to the development in [1], Eqs. (15) and (16) do not contain the "implicitness" factor $\alpha$. This factor was introduced in [1] to allow a semi-implicit extrapolation of the RHS source terms in Eqs. (15) and (16) to a time between $t^{n}$ and $t^{n+1}$. This was accomplished by approximating the $t^{n+1}$ value of a source term $S^{n+1}$ via

$$
S^{n+1} \approx S^{n}+\alpha \frac{\partial S}{\partial T} \Delta T .
$$

This approximation extrapolates $S$ to a time $t^{n}+\alpha t^{n+1}$, with $\alpha$ usually limited to the range [0.5, 1] for stability reasons [1] [4] [8]. Since standard practice in traditional IMC simulations is to use $\alpha=1$, we have not introduced it in the development in this paper. It would be relatively straightforward to do so by multiplying all of the extrapolated RHS derivative terms in Eqs. (15) and (16) by $\alpha$.

Solving Eq. (16) for $\Delta T$ results in

$$
\Delta T=\frac{\Delta t}{\rho c_{v}^{n}} \frac{\int \sigma^{n} I \mathrm{~d} \Omega \mathrm{d} v-\int \sigma^{n} B^{n} \mathrm{~d} \Omega \mathrm{d} v}{\left(1+\frac{\Delta t}{\rho c_{v}^{n}} \int\left[\sigma^{n} B^{\prime}-\sigma^{\prime}\left(I^{n}-B^{n}\right)\right] \mathrm{d} \Omega \mathrm{d} v\right)}
$$

Defining $g$ via

$$
g \equiv \frac{1}{1+\frac{\Delta t}{\rho c_{v}^{n}} \int\left[\sigma^{n} B^{\prime}-\sigma^{\prime}\left(I^{n}-B^{n}\right)\right] \mathrm{d} \Omega \mathrm{d} v},
$$

we can write Eq. (18) as

$$
\Delta T=\frac{\Delta t}{\rho c_{v}^{n}}\left[\int g \sigma^{n} I \mathrm{~d} \Omega \mathrm{d} v-\int g \sigma^{n} B^{n} \mathrm{~d} \Omega \mathrm{d} v\right] .
$$

The quantity $g$ reduces to $f$ defined by Eq. (10) if we ignore $\sigma^{\prime}$ and $B^{\prime}$, as the Fleck and Cummings algorithm does, and will be seen to play a role analogous to that of $f$ in the method being derived. 
Using Eq. (20) in Eq. (15) and disregarding the term that is quadratic in $\Delta T$ results in

$$
\begin{aligned}
\frac{1}{c} \frac{\partial I}{\partial t} & +\Omega \cdot \nabla I=-\sigma^{n} I+\sigma^{n} B^{n} \\
& -\frac{\Delta t}{\rho c_{v}^{n}}\left[\sigma^{n} B^{\prime}-\sigma^{\prime}\left(I^{n}-B^{n}\right)\right]\left[\int g \sigma^{n} I \mathrm{~d} \Omega \mathrm{d} v-\int g \sigma^{n} B^{n} \mathrm{~d} \Omega \mathrm{d} v\right] .
\end{aligned}
$$

First we will examine the term on the right-hand side of Eq. (21) involving an integral over the instantaneous value of the intensity, $I$. This term can be interpreted as a scattering term. We would like to cast it in a form that involves a probability distribution function $P_{s}(\Omega, v)$ times a magnitude independent of these variables. To get the probability distribution function, we normalize the function of $\Omega$ and $v$. Making use of the fact that

$$
\frac{\Delta t}{\rho c_{v}^{n}} \int\left[\sigma^{n} B^{\prime}-\sigma^{\prime}\left(I^{n}-B^{n}\right)\right] \mathrm{d} \Omega \mathrm{d} v=\frac{1-g}{g},
$$

we can write the term as

$$
P_{s}(\Omega, v) \frac{\Delta t}{\rho c_{v}^{n}} \int(1-g) \sigma^{n} I \mathrm{~d} \Omega \mathrm{d} v
$$

where

$$
P_{s}(\Omega, v) \equiv \frac{\sigma^{n} B^{\prime}-\sigma^{\prime}\left(I^{n}-B^{n}\right)}{\int\left[\sigma^{n} B^{\prime}-\sigma^{\prime}\left(I^{n}-B^{n}\right)\right] \mathrm{d} \Omega \mathrm{d} v}
$$

satisfies

$$
\int P_{s}(\Omega, v) \mathrm{d} \Omega \mathrm{d} v=1
$$

This allows us to identify $(1-g) \sigma$ as the effective scattering opacity, and $P_{s}$ as the probability distribution function for the scattered angle and frequency.

The remaining terms on the right-hand side of Eq. (21) represent thermal emission. As we did with the scattering term, we would like to separate this expression into a probability distribution function multiplied by a magnitude.

The thermal source term is

$$
\sigma^{n} B^{n}-\frac{\Delta t}{\rho c_{v}^{n}}\left[\sigma^{n} B^{\prime}-\sigma^{\prime}\left(I^{n}-B^{n}\right)\right] \int g \sigma^{n} B^{n} \mathrm{~d} \Omega \mathrm{d} v .
$$

We again normalize this function by integrating over $\Omega$ and $v$. Using the fact that

$$
\int \sigma^{n} B^{n} \mathrm{~d} \Omega \mathrm{d} v-\frac{\Delta t}{\rho c_{v}^{n}}\left[\int\left[\sigma^{n} B^{\prime}-\sigma^{\prime}\left(I^{n}-B^{n}\right)\right] \mathrm{d} \Omega \mathrm{d} v\right] \int g \sigma^{n} B^{n} \mathrm{~d} \Omega \mathrm{d} v=g \int \sigma^{n} B^{n} \mathrm{~d} \Omega \mathrm{d} v
$$

we obtain the following expression for the thermal emission source:

$$
P_{t}(\Omega, v) \int g \sigma^{n} B^{n} \mathrm{~d} \Omega \mathrm{d} v
$$

where

$$
P_{t}(\Omega, v) \equiv \frac{\sigma^{n} B^{n}-\frac{\Delta t}{\rho c_{v}^{n}}\left[\sigma^{n} B^{\prime}-\sigma^{\prime}\left(I^{n}-B^{n}\right)\right]}{\int g \sigma^{n} B^{n} \mathrm{~d} \Omega \mathrm{d} v}
$$


Using the definition of the Planck opacity, Eq. (5), and Eq. (4), we can write the total emission as $\operatorname{gc\sigma }_{P} a\left(T^{n}\right)^{4}$.

Combining these expressions, we can write Eq. (21), the semi-implicit version of the transport equation, as

$$
\begin{aligned}
\frac{1}{c} \frac{\partial I}{\partial t}+\Omega \cdot \nabla I(\sigma, v) & =\sigma^{n} I^{n+1} \\
& +P_{t}(\Omega, v) g \sigma_{P} c a\left(T^{n}\right)^{4} \\
& +P_{s}(\Omega, v) \int(1-g) \sigma^{n} I \mathrm{~d} \Omega^{\prime} \mathrm{d} v^{\prime}
\end{aligned}
$$

This equation may be compared to Eqs. (3.5) and (4.1a) in [1]. From this comparison, we see that the quantity $g$ acts in the same way as $f$ in the Fleck and Cummings IMC algorithm: it multiplies the absorption opacity and introduces an effective scattering.

The matter energy density can be updated by enforcing total energy conservation with Eq. (30). This results in

$$
\frac{\partial e_{m}}{\partial t}=\int g \sigma(v, T) I(v, \Omega) \mathrm{d} \Omega \mathrm{d} v-\int g \sigma(v, T) B(v) \mathrm{d} \Omega \mathrm{d} v
$$

This equation can also be derived by substituting the expression for $\Delta T$, Eq. (20), into Eq. (16).

\section{Approximations to $g, P_{s}$, and $P_{t}$ useful for numerical work.}

Eqs. (30) and (31) can be solved by the same numerical techniques used to solve the corresponding equations in [1]. However, the modified equations present two complications with respect to the original equations.

First, the emission and scattering probability distribution functions defined in Eq. (24) and Eq. (29) are functions of angle as well as frequency. In Fleck and Cummings IMC, probability distribution functions for both effective scattering and thermal emission are angle-independent and Planckian. That is, both are represented by the Planck function, Eq. (4).

The second complication is that we cannot ensure that $g$, defined by Eq. (19), satisfies $g>0$, while the "Fleck factor", defined by Eq. (10), is manifestly positive.

We will now discuss approximations for Eq. (24), Eq. (29) and Eq. (19) that mitigate these problems.

The angle dependence of Eqs. (24) and (29) results from the time $n$ value of the radiation intensity, because quantities such as $B$ and $\sigma$ are independent of angle. We could use tallies of the radiation intensity in the previous time step to give us a value for $I(\Omega, v)$ at time $n$. However, this would result in either values for the radiation intensity in a finite number of angle and energy bins, or some kind of functional representation of $I$ in $\Omega$ and $v$. Either would be subject to statistical noise.

The frequency dependence of the thermal source term is $\sigma^{n} B^{n}$ plus a correction factor in volving $\sigma^{\prime}$ multiplied by $I^{n}-B^{n}$. This term approximates the change in the emitted frequency due to the change in opacity over the time step. In the Fleck and Cummings algorithm, changes in the frequency of thermal emission over the time step are ignored. This approximation occurs in Eqs $(3.4 a-b)$ in [1], when the change in thermal emission over a time step is approximated by 
the change in radiation temperature over the time step. In effect,

$$
\begin{aligned}
\frac{\mathrm{d} B}{\mathrm{~d} T} & =\frac{\mathrm{d}\left(a T^{4} b\right)}{\mathrm{d} T} \\
& \approx \frac{\mathrm{d}\left(a T^{4}\right)}{\mathrm{d} T} b \\
& =4 a T^{3} b ;
\end{aligned}
$$

or, equivalently, $\frac{\mathrm{d} b}{\mathrm{~d} T} \approx 0$.

We do not want to calculate the angular dependence in $P_{t}(\Omega, v)$ as defined in Eq. (29) because of its susceptibility to statistical noise. We also note that the Fleck and Cummings algorithm is successful while employing the approximation in Eq. (32). For these two reasons we will use an approximation that is independent of angle:

$$
P_{t}(v) \approx \frac{\sigma^{n}\left(T^{n}\right) B\left(v, T^{n}\right)}{\int_{0}^{\infty} \sigma^{n}\left(T^{n}\right) B\left(v, T^{n}\right) \mathrm{d} v} .
$$

This is the same probability distribution function used in Fleck and Cummings.

Now we will turn to the probability distribution function for scattering, $P_{s}(\Omega, v)$ as defined in Eq. (24). The first term in the numerator of Eq. (24) is $\sigma^{n} B^{\prime}$. Using Eq. (32), we can approximate this as $\sigma^{n} 4 a T^{3} b(v, T)$. Ignoring the angular dependence and normalizing this expression gives

$$
P_{s}(\Omega, v) \approx \frac{\sigma^{n} B\left(v, T^{n}\right)}{\int_{0}^{\infty} \sigma^{n} B\left(v, T^{n}\right) \mathrm{d} v},
$$

which is the same probability distribution function used in Fleck and Cummings, and the same one we are employing for thermal emission.

Finally, we will turn to the expression for $g$, Eq. (19). This quantity reduces the thermal emission and replaces it with effective scattering, just as the quantity $f$ does in [1]. The integral term in the denominator of the definition of $\mathrm{g}$,

$$
\frac{\Delta t}{\rho c_{v}^{n}} \int \sigma^{n} B^{\prime}-\sigma^{\prime}\left(I^{n}-B^{n}\right) \mathrm{d} \Omega \mathrm{d} v,
$$

will contains two terms, one in $B^{\prime}$ and one in $\sigma^{\prime}$. Using Eq. (32), we can approximate the $B^{\prime}$ term, which lets us write

$$
\begin{aligned}
\frac{\Delta t}{\rho c_{v}^{n}} \int \sigma^{n} B^{\prime} \mathrm{d} \Omega \mathrm{d} v & \approx \frac{\Delta t}{\rho c_{v}^{n}} 4 a T^{3} c \int \sigma^{n} b \mathrm{~d} v \\
& \approx \frac{4 a T^{3}}{\rho c_{v}^{n}} \sigma_{P} \Delta t .
\end{aligned}
$$

Defining

$$
\beta \equiv \frac{4 a T^{3}}{\rho c_{v}^{n}}
$$

as in [1], we see we can approximate the first term in the denominator as $\beta c \sigma_{P} \Delta t$, which is the same quantity that appears in the denominator of the "Fleck factor" $f$ of Eq. (10). If we assume that $\sigma^{\prime}=0$, then $g=f$ 
The $\sigma^{\prime}$ term in Eq. (19) has the effect of increasing the effective scattering when the opacity decreases as a function of time and the material is going to absorb energy from the radiation and increase in temperature. If $\sigma^{\prime}<0$, which is usually the case, and $I^{n}>B^{n}$, then $-\sigma^{\prime}\left(I^{n}-B^{n}\right)>0$, and $g<f$. This decreases the absorption and increases the effective scattering relative to the Fleck and Cummings method. If the material is going to heat up during the time step, and its opacity decreases with $T$, it should not absorb as much energy as if the opacity is constant. Thus, this behavior is physically reasonable and we can conjecture that problems in which a material with variable opacity is heated by radiation will show less unphysical overheating than we find with Fleck and Cummings IMC. In the next section, we will see that that is indeed the case.

The term

$$
\int \sigma^{\prime}\left(I^{n}-B^{n}\right) \mathrm{d} \Omega \mathrm{d} v
$$

is a weighted approximation to $\sigma^{\prime}$. Rather than use a tally over $I^{n}$ to calculate it, we make the approximation

$$
I^{n} \approx \frac{c a T_{r}^{4} b\left(v, T^{n}\right)}{4 \pi}
$$

where the radiation temperature is defined via

$$
T_{r} \equiv \frac{1}{c} \int I^{n} \mathrm{~d} \Omega \mathrm{d} v
$$

Note that we are using Eq. (39) only in the calculation of $g$. This value for $I^{n}$ allows us to make the following approximation:

$$
\int \sigma^{\prime} I^{n} B^{n} \mathrm{~d} \Omega \mathrm{d} v \approx c \sigma_{P}^{\prime}\left(a T_{r}^{4}-a T^{4}\right) .
$$

Here we have also assumed $\frac{\mathrm{d} b}{\mathrm{~d} T} \approx 0$ in the derivative of the Planck opacity, as in Eq. (32).

These (admittedly severe) approximations allow us to use the following definition for the modified "Fleck factor" $g$ :

$$
g=\frac{1}{1+\beta c \sigma_{P} \Delta t-c \sigma_{P}^{\prime}\left(a T_{r}^{4}-a T^{4}\right) \frac{\Delta t}{\rho c_{v}^{n}}}
$$

As noted, we can have $g<0$ if $\sigma_{P}^{\prime}\left(a T_{r}^{4}-a T^{4}\right)$ is sufficiently large and positive, resulting in a negative absorption opacity. While difficult to simulate, this is not actually unphysical. If we had $\sigma_{P}^{\prime}<0$ and $T>T_{r}$, then energy should be transferred from the material to the radiation field. A negative absorption would do this by having photons increase in energy as they propagated, taking the energy from the material and lowering its temperature. In effect, the photons deposit a negative energy into the material. This is the physically correct direction for energy flow. However, the absorption opacity appears in an exponential when calculating energy deposition. Using a negative absorption opacity causes a large risk of an overflow or a negative matter temperature. So in practice, we have used the following expression for $g$ :

$$
g=\frac{1}{1+\beta c \sigma_{P} \Delta t-\min \left[\sigma_{P}^{\prime}\left(a T_{r}^{4}-a T^{4}\right) \frac{c \Delta t}{\rho c_{v}^{n}}, 0\right]}
$$

This form for $g$ ensures that we will always have $g \geq f$, so we will always have more effective scattering and hence a lower effective absorption when $g$ replaces $f$. There are cases 
where increasing the effective absorption would actually be physically reasonable. For example, if $\sigma_{p}^{\prime}<0$ and $T>T_{r}$, then increasing the absorption would bring the matter and radiation in the simulation into equilibrium more rapidly. In this case, the extra term in Eq. (43) would be positive, would result in $g<f$, and would increase the effective absorption. However, we prefer to define $g$ such that $g \geq f$ for the following reason. In radiation-hydrodynamics simulations, zones which become unphysically hot when $T_{r}>T$ can cause spurious features such as shocks to form if they rapidly increase in temperature. Zones with $T>T_{r}$ and which cool too slowly are unlikely to cause shocks to form. Although allowing $g<f$ might address accuracy issues in those zones, we prefer to use the more stringent limit embodied in Eq. (43) and maintain $g \geq f$ at all times.

Eqs. (30) and (31), with $g$ defined by Eq. (43), $P_{s}$ defined by Eq. (24) and $P_{t}$ defined by Eq. (29), are a set of equations that can be solved by the same techniques as those employed to solve the equations of [1]. If we assume $\sigma^{\prime}=0, g=f$ and we recover the results of [1].

When the opacity $\sigma$ has a simple analytic form, $\sigma_{P}^{\prime}$ can be calculated directly. For complicated forms, or for tabular data, $\sigma_{P}^{\prime}$ can be approximated with a difference form. For example,

$$
\sigma_{P}^{\prime} \approx \frac{\sigma_{P}\left(\rho^{n}, 1.001 T^{n}\right)-\sigma_{P}\left(\rho^{n}, T^{n}\right)}{0.001 T^{n}}
$$

This approximation was used for the second test problem in the next section, which employed a tabular multigroup opacity.

Now that we have defined the new method, it is useful to compare it with several other recent attempts to modify IMC. The algorithm described in this paper (henceforth referred to as "Modified IMC") attempts to improve the accuracy of a Monte Carlo simulation by improving the accuracy of the value of the opacity used in the time step. It does this by using information about the derivative of the opacity at the beginning of the time step. Cheatham [9] also describes a modified IMC algorithm that attempts to get a more accurate value of the opacity. That algorithm does this by a predictor-corrector methodology. An IMC simulation is run to get an estimate of the future matter temperature $T\left(t^{n+1}\right)$. This is the predictor step. The estimate of $T\left(t^{n+1}\right)$ is then used to get an updated value of the opacity for another IMC simulation, the corrector step, the results of which are used as the final $t^{n+1}$ values. Compared to Modified IMC, Cheatham's method has the advantage of getting an estimate of the opacity using a calculation of the $t^{n+1}$ temperature, which presumably makes it more accurate than an extrapolation based on the $t^{n}$ derivative. However, it has the drawback that two IMC simulations must be run for each time step.

The Modified IMC method attempts to reduce overshoots in $T$ by reducing the effective absorption by replacing the standard "Fleck factor" $f$ with a function that is smaller. It shares this characteristic with the method described by McClarren and Urbatsch [10]. In this work, the authors solve linearized versions of Eqs. (1) and (6) using an integrating factor rather than by assuming that the emission and absorption are constant during the time step. This results in the replacement of $f$ by a factor

$$
m_{\infty} \equiv \exp \left[-\beta \sigma_{P} c \Delta t\right]
$$

where all values are $t^{n}$ values. Using this quantity rather than $f$ in a Monte Carlo simulation can reduce overheating. However, it can lead to values of effective absorption that are too low. In this case, zone temperatures change at an unphysically slow rate. In this case, $m_{\infty}$ is replaced by an expansion of the exponential, with the order of the expansion determined by a function of $T$ and $T_{r}$ (see [10] for details). 
In [11], Wollaber replaces $f$ with a time-dependent factor, obtained by applying an integrating factor to linearized versions of Eqs. (1) and (6). This is done to improve the temporal accuracy of the IMC method to second order when $\Delta t$ is small. This change leaves large time step behavior, such as overheating, largely unchanged.

The methods described in [9], [10], and [11] all use linearized versions of the transport and matter energy equations that use the approximation Eq. (32) to approximate the thermal source term. This approximation ignores $\sigma_{P}^{\prime}$. The linearized equations used in these methods could be replaced by Eqs. (15) and (16). This would introduce terms containing $\frac{\sigma_{P}}{d T}{ }^{n}$, resulting in a combination of the Modified IMC method and the methods of [9], [10], and [11]. Combining the different methods is beyond the scope of this paper.

\section{Numerical Results}

In this section, we will show numerical results for two test problems using both the original Fleck and Cummings algorithm, and the modified IMC algorithm where $f$ is replaced by $g$. These simulations were performed using the IMC package in the Kull code [12].

The first test is an infinite medium problem, similar to that described in [13], but with a temperature-dependent opacity. The matter has an absorption opacity of the form $\sigma(T)=$ $\sigma_{0} T^{-5}$ and a constant heat capacity. We use the values $\sigma_{0}=10^{-3} \mathrm{~cm}^{-1} \mathrm{keV}^{-5}$ and $c_{v}=$ $5 \times 10^{14} \mathrm{erg} /(\mathrm{g} \mathrm{keV})$. We take the initial values $T=0.01 \mathrm{keV}$, and $T_{r}=1.46512 \mathrm{keV}$. These values will lead to a final equilibrium temperature of $T=1.0 \mathrm{keV}$ after about $2 \times 10^{-8} \mathrm{sec}$. The density is constant at $1.0 \mathrm{~g} / \mathrm{cm}^{-3}$ This problem, like the one presented in [13], has a semi-analytic solution. The test problem and its solution are described in more detail in the appendix.

The test problem begins with $T_{r}>T$. The opacity has an initial value of $10^{7} \mathrm{~cm}^{-1}$. This very large value will cause a large amount of energy to be absorbed, unless the time step is very small. The heat capacity is low, relative to $a T^{3}$, so the matter temperature will increase by a large amount compared to the radiation temperature when energy is absorbed. Because $\sigma$ is large and $c_{v}$ is small, we would expect IMC simulations of this problem to demonstrate overheating unless the time step used is very small compared to the equilibration time.

We will present results for this test problem using both the standard Fleck and Cummings IMC algorithm and the modified IMC algorithm. The Fleck and Cummings IMC algorithm will be seen to overshoot unless very small time steps are taken, while the modified IMC algorithm will not, even when very large values of $\Delta t$ are used.

Fleck and Cummings IMC results are displayed in Fig. 1. This plot shows matter and radiation temperature vs time for IMC simulations with constant $\Delta t$, for $\Delta t$ varying between $10^{-21}$ and $10^{-9} \mathrm{sec}$. Matter temperature $T$ is plotted in red, and radiation temperature $T_{r}$ in blue. Each IMC simulation is represented by a pair of lines, one red and one blue. The lines for each simulation begin at a time equal to the value of $\Delta t$ used in the simulation. That is, the first point plotted for any simulation is the value at the end of the first time step; the $t=0$ initial conditions are not plotted. Some simulations with very small time steps were not run to completion, because they would have taken a prohibitive amount of computer time. This is why the red and blue lines that begin at $t=10^{-18}$ and $t=10^{-17}$ end at $t=10^{-14}$. The analytic answer for both $T$ and $T_{r}$ is in black.

The results of the IMC simulations in Fig. 1 depend very strongly on the value of $\Delta t$. The initial conditions of the simulation have $T_{r}(t=0)>T(t=0)$, and we should have $T_{r} \geq T$ at all subsequent times [4] [8]. We find that IMC simulations must have a very small $\Delta t$ to satisfy this requirement. 
First, let us examine the results for $\Delta t=10^{-20} \mathrm{sec}$. These are represented by the red and blue lines beginning at $t=10^{-20} \mathrm{sec}$. We see that the value of $T$ after the end of the first time step obtained in this simulation is slightly greater than the analytic value, and the value of $T_{r}$ in the first time step is slightly lower than the analytic value. In subsequent time steps, the results of the IMC simulation move towards the analytic results, so that by $t=10^{-19} \mathrm{sec}$ the simulation results are indistinguishable from the analytic answer.

For $\Delta t=10^{-19} \mathrm{sec}, T$ is larger than the semi-analytic answer, but remains lower than $T_{r}$, after the first time step. The large $T$ obtained after the first time step leads to a small absorption opacity, so $T$ remains almost constant after the first time step, until it eventually approaches the semi-analytic answer after $t=10^{-16} \mathrm{sec}$. For $\Delta t=10^{-18} \mathrm{sec}, T$ increases by a larger amount, reaching a value of $0.34 \mathrm{keV}$, and remaining constant after that. This is still less than the value of $T_{r}$ for this simulation, which is $1.36 \mathrm{keV}$. For $\Delta t=10^{-17} \mathrm{sec}, T$ reaches a value of $1.21 \mathrm{keV}$, greater than the value of $T_{r}$, which is $0.69 \mathrm{keV}$. This is a violation of thermodynamic consistency. For this simulation, the red line representing matter temperature is above the associated blue line representing the radiation temperature. As with the simulations using $\Delta t=10^{-19} \mathrm{sec}, \Delta t=10^{-18}$ $\mathrm{sec}$, and $\Delta t=10^{-17} \mathrm{sec}$, the erroneously large value of $T$ remains constant in time because of the low value of $\sigma$ that occurs when the temperature is large.

For all simulations with values of $\Delta t>10^{-17} \mathrm{sec}$, essentially all the radiation energy is absorbed by the material in the first time step. The matter temperature jumps to a value of $1.21 \mathrm{keV}$ after one time step. This is the value of $T$ obtained when all of the energy in the problem is in matter internal energy, there is no radiation energy, and the radiation temperature drops to essentially zero. For these simulations, the blue lines representing radiation temperature begin near zero, and slowly rise up toward the analytic solution as the simulation progresses. The red lines representing matter temperature are all near $1.21 \mathrm{keV}$, and remain there until the simulations reach equilibrium. In these simulations, equilibrium is reached later than it is in the analytic answer.

(For the run with $\Delta t=10^{-17} \mathrm{sec}, T_{r}$ has a value of $1.1 \mathrm{keV}$ after the first time step. $T_{r}$ then drops to the value of $0.69 \mathrm{keV}$. The reason for this is due to the method of calculating $T_{r}$. $T_{r}$ is calculated from an average of $e_{r}$ over every path, rather than summing the energy of every photon that reaches census. This has the effect of time centering $T_{r}$ in the middle of the time step, rather than the end. So in the first time step, $T_{r}$ takes a value, $1.1 \mathrm{keV}$, intermediate between the initial value of $1.46512 \mathrm{keV}$ and the final value of $0.69 \mathrm{keV}$. The sum of the energy density of the census photons after 1 time step has a value which satisfies $\left(e_{r}(\Delta t) / a\right)^{0.25}=0.69 \mathrm{keV}$. So the value of $1.1 \mathrm{keV}$ after 1 time step is a transient artifact, rather than a physical effect.)

Fig. 1 may be compared to Fig. 4 of [8]. That figure shows damped oscillatory behavior in an IMC simulation of an infinite medium test problem with constant opacity. This oscillatory behavior occurs for the same reason that overshooting of the analytic temperature occurs for larger time steps in Fig. 1. Initially, $T_{r}(t=0)>T(t=0)$. The test problem has a large value of $\sigma$ and a "Fleck factor" satisfying $f \approx 1$, leading to almost complete absorption of the radiation by the matter in the first time step. This causes the value of $T_{r}$ in the next time step to fall to a small value, and the matter temperature to rise to a large value, such that $T_{r}(t=\Delta t)<T(t=\Delta t)$. On the second time step, the large value of $T$ causes a large amount of thermal emission, leading to $T_{r}(t=2 \Delta t)>T(t=2 \Delta t)$. The test problem shown in Fig. 1 behaves in a similar manner in the first time step. It does not exhibit oscillatory behavior in subsequent time steps because the temperature-dependent opacity drops to a very small value when $T(t=\Delta t)$ becomes large. Since $\sigma_{P} T^{4}(t=\Delta t)$ is small, there is very little thermal emission, and $T$ remains at the large value of $T(t=\Delta t)$. 
Fleck \& Cummings solutions with various $\mathrm{dt}$

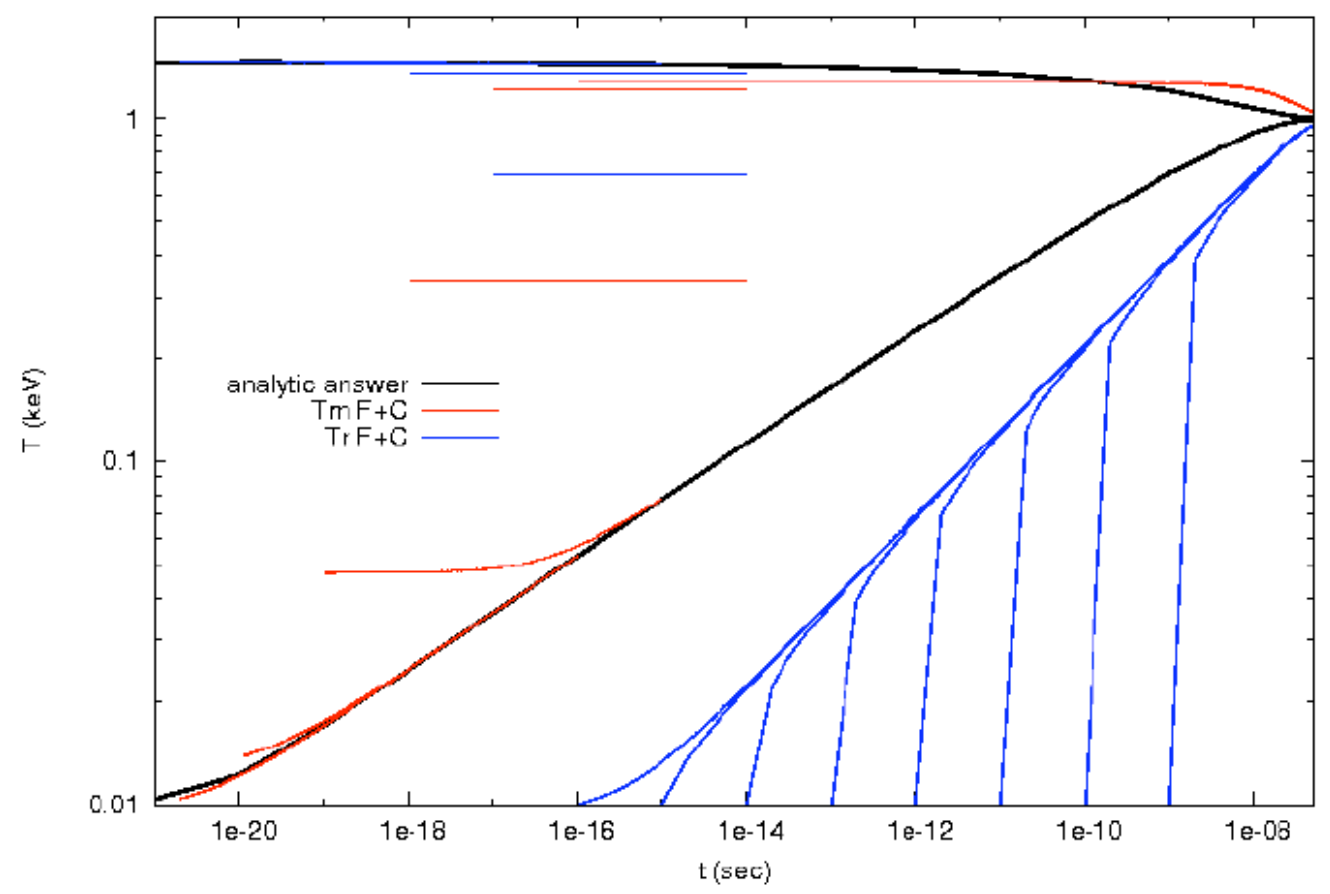

Figure 1: Fleck and Cummings IMC results for several different values of $\Delta t$ for the $\sigma \sim T^{-5}$ test problem described in the appendix. Matter and radiation temperature are plotted vs. time. Matter temperature is denoted by $T_{m}$ and radiation temperature by $T_{r}$.

Results using the Modified IMC algorithm are displayed in Figure 2. The results are similar for all values of $\Delta t$. In all cases, $T$ remains below both $T_{r}$ and the semi-analytic value. The modified IMC algorithm does not overshoot, even for values of $\Delta t$ more than 10 orders of magnitude larger than the value of $\Delta t$ for which Fleck and Cummings IMC overshoots. Even for $\Delta t=10^{-7} \mathrm{sec}$, which is a significant fraction of the equilibration time of $\sim 2 \times 10^{-8} \mathrm{sec}$, the modified IMC algorithm keeps $T<T_{r}$, and reaches the correct asymptotic value $\left(T=T_{r}=1.0\right.$ $\mathrm{keV})$ at approximately the correct time.

The reason for the difference in behavior of the two methods is shown in Table 1. The "Fleck factor" $f \approx 1$ even for the smallest value of $\Delta t$ used, so the amount of effective scattering introduced by the IMC algorithm is insignificant. The absorption opacity $f \times \sigma\left(T_{0}\right) \approx \sigma\left(T_{0}\right)=$ $10^{7} \mathrm{~cm}^{-1}$. Since the absorption opacity is very large, a significant fraction of the radiation energy is absorbed by the matter, and the temperature increases. For $\Delta t>10^{-17} \mathrm{sec}$, almost all the radiation energy is absorbed by the matter in one time step, which causes $T_{r}$ to drop to a very low value. Since the opacity is very low for large $T$, the hot matter radiates very little energy, and $T$ remains large.

In contrast, the modified "Fleck factor" $g$ has a small value, even for very large $\Delta t$. The amount of effective scattering is very large, and the effective absorption opacity is very small. So the amount of energy absorbed from the radiation by the matter is much smaller than that 


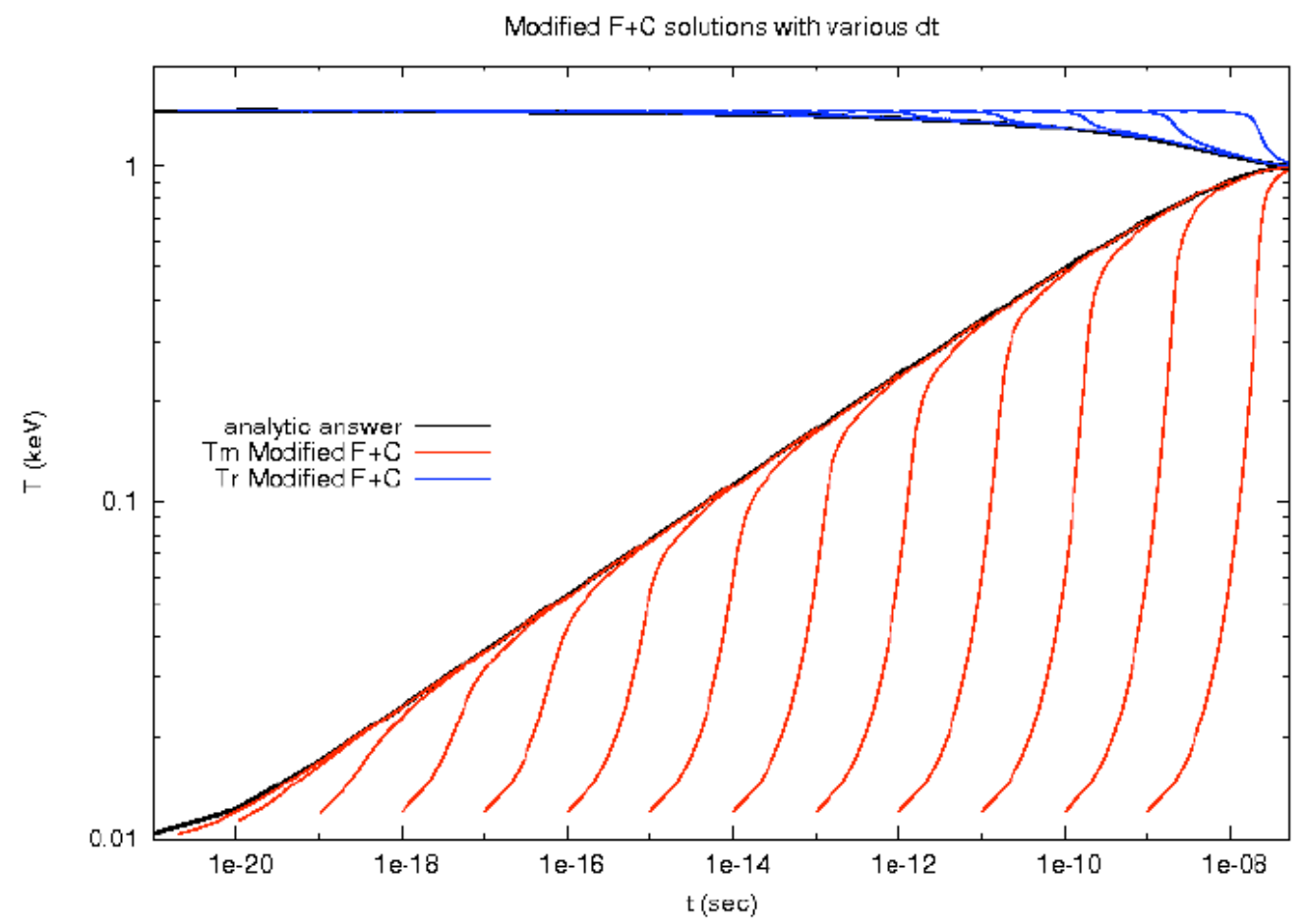

Figure 2: Modified IMC results for several different values of $\Delta t$ for the $\sigma \sim T^{-5}$ test problem described in the appendix. Matter and radiation temperature are plotted vs. time. Matter temperature is denoted by $T_{m}$ and radiation temperature by $T_{r}$.

absorbed during Fleck and Cummings IMC simulation. The matter temperature remains low, the opacity remains high, and $T$ slowly increases to the value obtained from the semi-analytic solution.

Since the effective scattering is very large in the modified IMC algorithm, particles will have a very small mean free path, and simulations can take a long time. In these calculations, we have used the "random walk" algorithm described in [14]. This speeds up the calculation significantly by using a solution of the diffusion equation to move particles to positions that represent the effects of many scatters. As a result of employing the "random walk" algorithm in simulations using the modified IMC algorithm, all simulation of the first test problem described here took on the order of a few minutes using one Intel processor running the Linux operating system.

This test problem can also be simulated with diffusion. Since the problem has no spatial variation, the differences in the spatial discretization of diffusion and Monte Carlo will not affect the answer. This allows us to use the diffusion simulation to examine the behavior of a different temporal discretization than the one used in either Monte Carlo algorithm. The diffusion simulation used the flux-limited diffusion package of the Kull code [12]. This package iterates on the matter temperature used in the thermal emission source term of the transport equation, but uses a value of the opacity at the beginning of the time step. In effect, the diffusion temporal discretization uses the $t^{n+1}$ value of the matter temperature but the $t^{n}$ value of the opacity. 
Table 1: Values of opacity, f and g with $T=0.01 \mathrm{keV}$ and $T_{r}=1.46512 \mathrm{keV}$ for values of $\Delta t$ used in the first test problem.

\begin{tabular}{|l|l|l|}
\hline$\Delta t(\mathrm{sec})$ & $f(T)$ & $g\left(T, T_{r}\right)$ \\
\hline $10^{-19}$ & 1.000000000000 & 1 \\
$10^{-18}$ & 1.000000000000 & 0.999998 \\
$10^{-17}$ & 1.000000000000 & 0.999981 \\
$10^{-16}$ & 1.000000000000 & 0.999811 \\
$10^{-13}$ & 0.999999999671 & 0.840671 \\
$10^{-11}$ & 0.999999967095 & 0.0501186 \\
$10^{-9}$ & 0.999996709468 & 0.000527352 \\
\hline
\end{tabular}

The results of simulations using the diffusion algorithm are displayed in Figure 3. Various values of $\Delta t$ from $10^{-19}$ to $10^{-13}$ seconds were used. For $\Delta t=10^{-19} \mathrm{sec}, T$ jumps from the initial value of $0.01 \mathrm{keV}$ to $0.05 \mathrm{keV}$. This reduces the opacity to a smaller value, reducing absorption by the matter, and the solution remains constant, until it begins to approach the analytic answer at around $t=5 \times 10^{-15} \mathrm{sec}$. When $\Delta t$ is increased to $10^{-18} \mathrm{sec}, T$ jumps to the higher value of $\sim 0.3 \mathrm{keV}$. For $\Delta t=10^{-16} \mathrm{sec}$, the solution jumps to a value of $T$ slightly lower than $1.0 \mathrm{keV}$, and a value of $T_{r}$ that is slightly less. For $\Delta t=10^{-15} \mathrm{sec}$, the solution jumps to the equilibrium value, $T=T_{r}=1.0 \mathrm{keV}$, remaining constant thereafter. (The number of time steps was limited to 5000, which is why the lines in Figure 3 end.)

The behavior of the diffusion simulations show some similarities to the behavior of the Fleck and Cummings IMC solution. Unless a very small time step is used, the simulation results in matter temperature that is too large and a radiation temperature that is too small. Unless $\Delta t$ is less than about $10^{-16} \mathrm{sec}$, the diffusion simulation jumps immediately to the equilibrium temperature.

The behavior of the diffusion algorithm is more accurate than that of IMC for large time steps, because the diffusion solution remains thermodynamically consistent, with $T_{r}>T$, while IMC produces results with $T>T_{r}$. This occurs because the diffusion simulation iterates on the matter temperature used for thermal emission. This iterative procedure means that emission in time step $n$ is proportional to $T^{4}$ at $t^{n+1}$, rather than the estimate $f T^{4}$ at $t^{n}$ that IMC uses. However, the diffusion simulation still uses $\sigma$ at $t^{n}$ to calculate thermal emission. In effect, the diffusion simulation is implicit in temperature, but explicit in opacity.

The Modified IMC algorithm produces more accurate answers for this test problem than diffusion, even with much larger $\Delta t$. For this test problem, with an opacity that has a strong temperature dependence, a more accurate answer is obtained by an algorithm that is semi-implicit in both $T$ and $\sigma$ than by an algorithm that is fully implicit in $T$ but explicit in $\sigma$.

The second test problem is a coupled radiation-hydrodynamics test problem with tabular multigroup opacities and a tabular equation of state. The problem consists of a slab of silicon dioxide with a density of $0.01 \mathrm{~g} \mathrm{~cm}^{-3}$ and a slab of plastic doped with bromine with a density of $0.5 \mathrm{~g} \mathrm{~cm}^{-3}$. Both materials have a temperature of $0.025 \mathrm{keV}$. The plastic occupies $x=[0,5 \mathrm{~cm}]$ and the silicon dioxide occupies $x=[5,10 \mathrm{~cm}]$. A radiation source characterized by temperature of $1 \mathrm{keV}$ is applied to the face at $x=10 \mathrm{~cm}$.

The mesh for the problem uses 200 equal zones. The opacities of both materials were modeled with 14 groups with boundary values of $[0.0001,0.01,0.05,0.25,0.5,0.75,1.0,1.25,2.0$, $5.0,10.0,15.0,20.0,25.0,30.0] \mathrm{keV}$.

The temperature source applied to the silicon dioxide drives a Marshak wave through it and into the plastic. As with the infinite medium test problem described above, we can get overheat- 


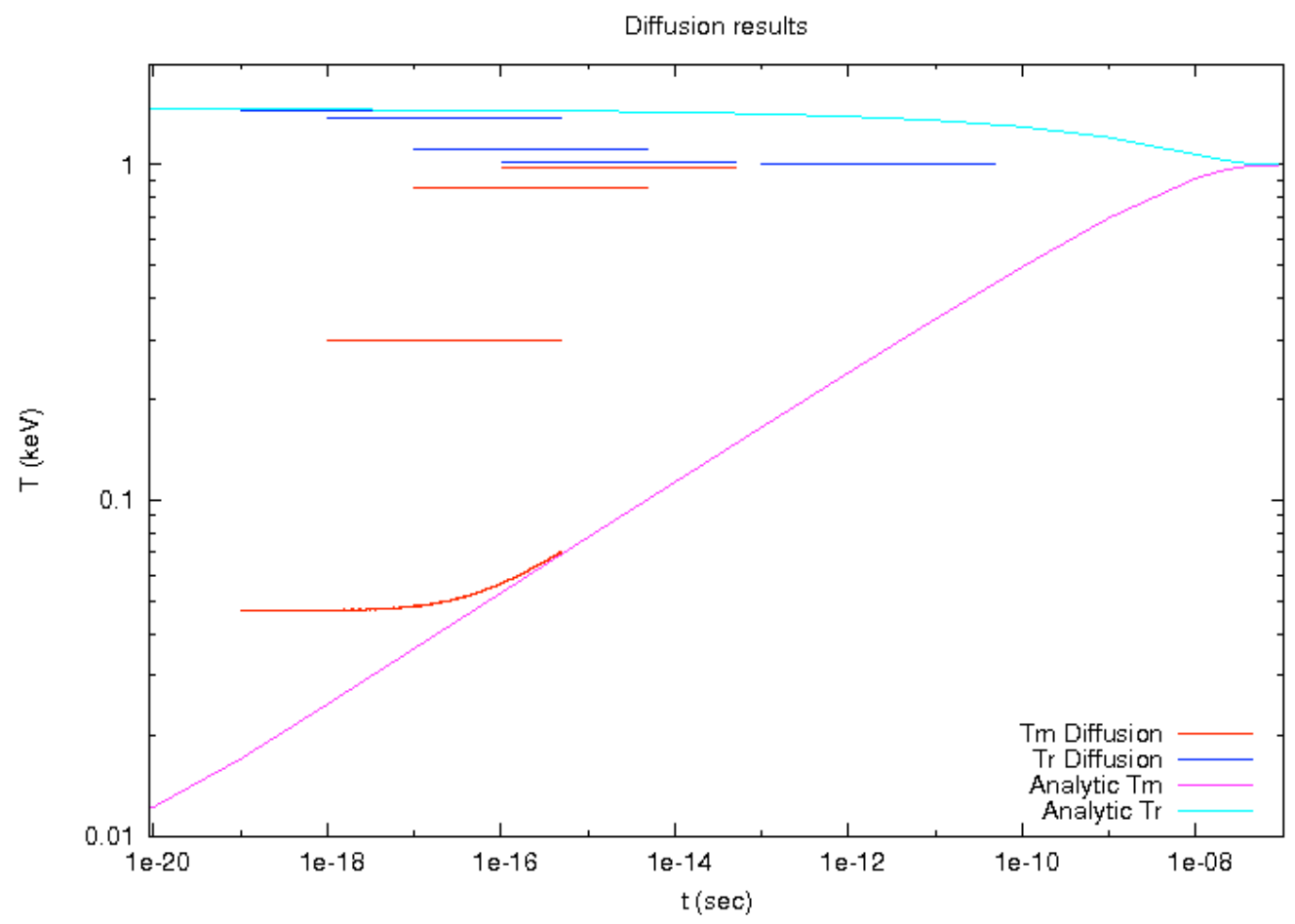

Figure 3: Diffusion results for 5 different values of $\Delta t$ for the $\sigma \sim T^{-5}$ test problem described in the appendix. Matter and radiation temperature are plotted vs. time. Matter temperature is denoted by $T_{m}$ and radiation temperature by $T_{r}$.

ing in the material when $T_{r}>T$. Because the problem uses multigroup opacities, high frequency photons see a lower opacity and have a larger mean free path than low frequency photons. These high frequency photons can penetrate into the material ahead of the Marshak wave and raise the radiation temperature there. Since $g$ can be lower than $f$ when $T_{r}>T$ because of the last term in the denominator in Eq. (43), we expect that problems with overheating might be reduced by the modified IMC algorithm. This proves to be the case.

Figure 4 shows results for this test problem using both Fleck and Cummings IMC and the Modified IMC method at a simulation time of $1.0 \times 10^{-7} \mathrm{sec}$. Both simulations used the same value of $\Delta t=1.0 \times 10^{-11} \mathrm{sec}$. At the plotted time, the Marshak wave has proceeded through the silicon dioxide and is entering the plastic. Multigroup effects cause the radiation temperature in the plastic to be higher than the material temperature in the plastic.

The Fleck and Cummings IMC simulation clearly shows unphysical overheating near the temperature source at $\mathrm{x}=10 \mathrm{~cm}$. The material near the source should be at a temperature slightly lower than the source temperature, $1 \mathrm{keV}$, but has reached a temperature of almost $10 \mathrm{keV}$. Some regions of material further from the source have $T>1 \mathrm{keV}$ as well.

In contrast, the modified IMC method has $T<1 \mathrm{keV}$ everywhere; there is no sign of overheating. In the silicon dioxide, $T$ is smooth, and everywhere is slightly lower than $T_{r}$, which has a value approximately the same as the source temperature, $1 \mathrm{keV}$.

Lowering the value of $\Delta t$ used in the Fleck and Cummings IMC simulation eliminates the 


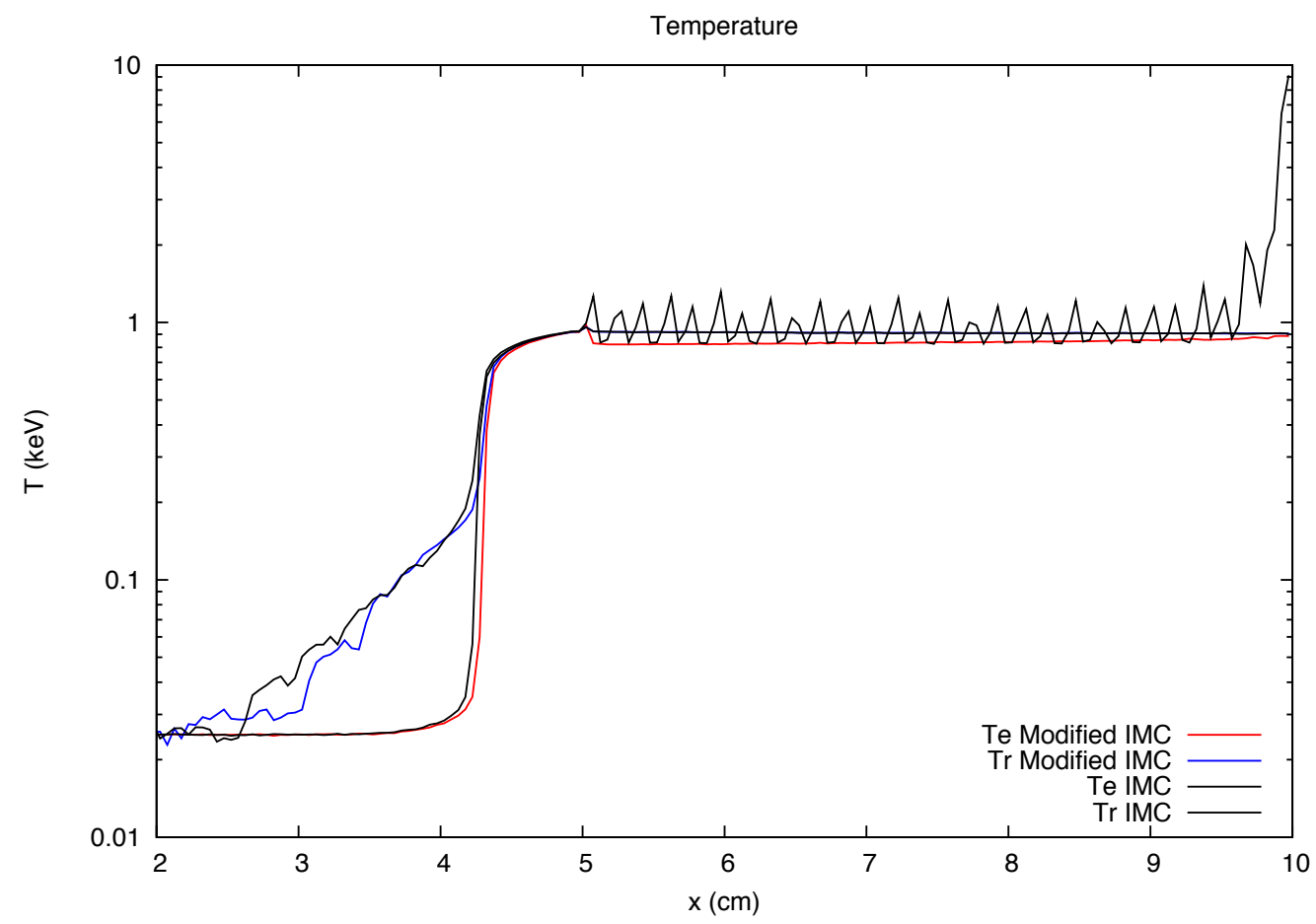

Figure 4: Fleck and Cummings IMC and modified IMC results for the radiation-hydrodynamics test problem. Matter and radiation temperature are plotted vs. space at $\mathrm{t}=1.0 \times 10^{-7} \mathrm{sec}$. Both simulations used $\Delta t=10^{-11} \mathrm{sec} . T_{e}$ denotes electron temperature and $T_{r}$ denotes radiation temperature.

overheating observed in Figure 4. Experimentation shows that the spike in the temperature of the silicon dioxide near the source is eliminated when $\Delta t=1.25 \times 10^{-12} \mathrm{sec}$ is used. With this value of $\Delta t$, Fleck and Cummings IMC gets results similar to those obtained by the modified IMC method with $\Delta t=10^{-11} \mathrm{sec}$. Figure 5 compares the Fleck and Cummings IMC simulation using $\Delta t=1.25 \times 10^{-12} \mathrm{sec}$ to the modified IMC simulation using $\Delta t=10^{-11} \mathrm{sec}$. The enhanced stability of the modified IMC method on this test problem is demonstrated by the fact that it produces results with no overheating with a value of $\Delta t$ that is 8 times larger than that needed by Fleck and Cummings IMC.

(The larger $\Delta t$ used in the modified IMC method is the reason that it produces smoother results for $T$ and $T_{r}$ in Fig. 5 in the cold region ahead of the Marshak wave. Cold regions typically have fewer particles because the number of thermally emitted particles is proportional to $T^{4}$. When $\Delta t$ is larger, more particles can travel between zones. This means that the temperature of the zone is calculated with better statistics, and is hence smoother.)

The reason that the anomalous high matter temperatures seen by the Fleck and Cummings IMC simulations in Figure 4 persist is shown in Figure 6. This plot depicts the Planck opacity for the two simulations depicted in Figure 4. The unphysically hot regions of silicon dioxide have a very low $\sigma_{P}$, reducing the rate of thermal emission. The unphysically hot material does not cool as a result. This behavior is similar to that observed in simulations in Figure 1 when larger 


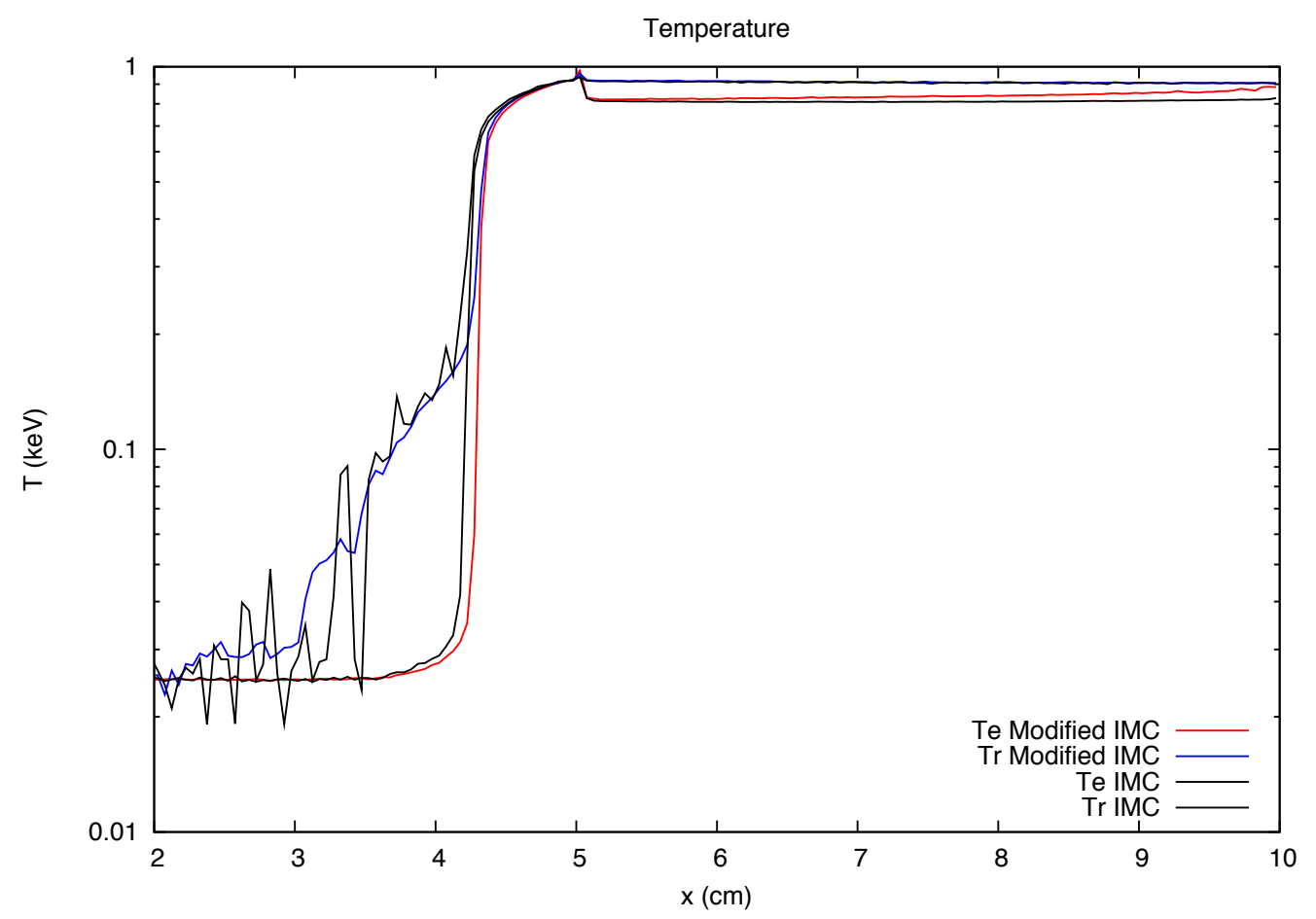

Figure 5: Fleck and Cummings IMC and Modified IMC results for the radiation-hydrodynamics test problem. Matter and radiation temperature are plotted vs. space at $\mathrm{t}=1.0 \times 10^{-7} \mathrm{sec}$. Fleck and Cummings IMC used $\Delta t=1.25 \times 10^{-12} \mathrm{sec}$. The modified IMC method used $\Delta t=10^{-11} \mathrm{sec} . T_{e}$ denotes electron temperature and $T_{r}$ denotes radiation temperature.

values of $\Delta t$ are used.

Figure 7 shows the values of $f$ and $g$ for the Fleck and Cummings IMC and modified IMC simulations of the radiation hydrodynamics test problem. Both simulations used $\Delta t=10^{-11} \mathrm{sec}$. The higher radiation temperature in front of the Marshak wave causes $g<f$. The value of $g$ reaches a low of 0.1 at the front of the Marshak wave in the plastic, while the lowest value of $f$ is 0.5. The absorption opacity is proportional to $f$ in Fleck and Cummings IMC and $g$ in modified IMC. So the particles in the modified IMC simulation are losing energy to the matter at a rate that is 5 times lower than that of the Fleck and Cummings IMC simulation. This lowered rate of coupling between the matter and radiation reduces the production of unphysically high matter temperatures.

\section{Conclusions}

We develop a modification of the "Fleck factor" in the IMC radiation transport method. This modification is developed by a semi-implicit temporal discretization of the thermal emission term in the transport equation. It takes into account the change in the opacity with temperature during a time step, and is sensitive to the difference between radiation and matter temperature. It has the effect of increasing the amount of effective scattering, and thus decreasing the absorption, 


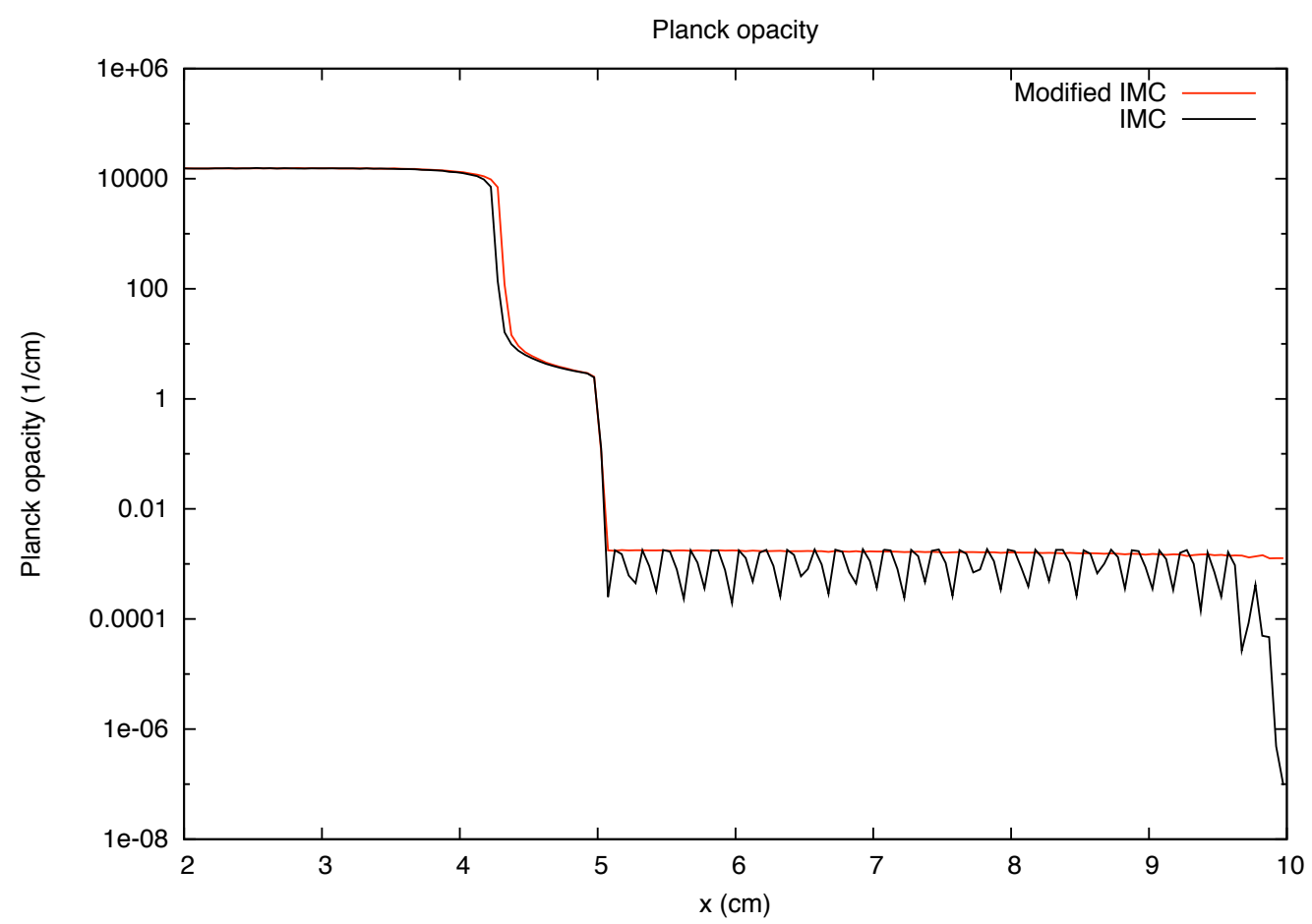

Figure 6: Opacity vs. space at $\mathrm{t}=1.0 \times 10^{-7} \mathrm{sec}$. for Fleck and Cummings IMC and modified IMC simulations of the radiation-hydrodynamics test problem. Both simulations used $\Delta t=10^{-11} \mathrm{sec}$.

for simulations with temperature-dependent opacities where the radiation temperature is greater than the matter temperature. The lower amount of absorption reduces the tendency for IMC simulations to produce unphysically large matter temperatures in some simulations.

\section{Appendix: semi-analytic solution of infinite medium test problem with temperature- dependent gray opacity.}

Here we present a semi-analytic solution of an infinite medium test problem with temperaturedependent gray opacity. This test problem is very similar to the constant opacity test problem presented in [13], and the solution technique is essentially the same as the one described there. This version of the test problem with a temperature-dependent opacity was first described in [7].

The test problem has a constant density $\rho$, a constant heat capacity $c_{v}$, and an absorption opacity $\sigma(T)=\sigma_{0} T^{-n}$, with $n$ a non-negative integer. The test problem has no radiation source. The solution of the test problem is obtained by solving Eqs. (1) and (6) with these assumptions.

The assumption of constant $c_{v}$ allows us to replace $e_{m}$ with temperature via the equation of state $e_{m}=\rho c_{v} T$. The fact that there are no sources in the problem means that the total energy $e_{t} \equiv e_{r}+e_{m}$ is a constant, determined by the initial radiation and matter temperatures. This allows us to eliminate the radiation energy density $e_{r}$ in terms of $e_{t}$ and $T$ via $e_{r}(t)=e_{t}-\rho c_{v} T(t)$. Eqs. 
Fleck factor and Modified Fleck factor

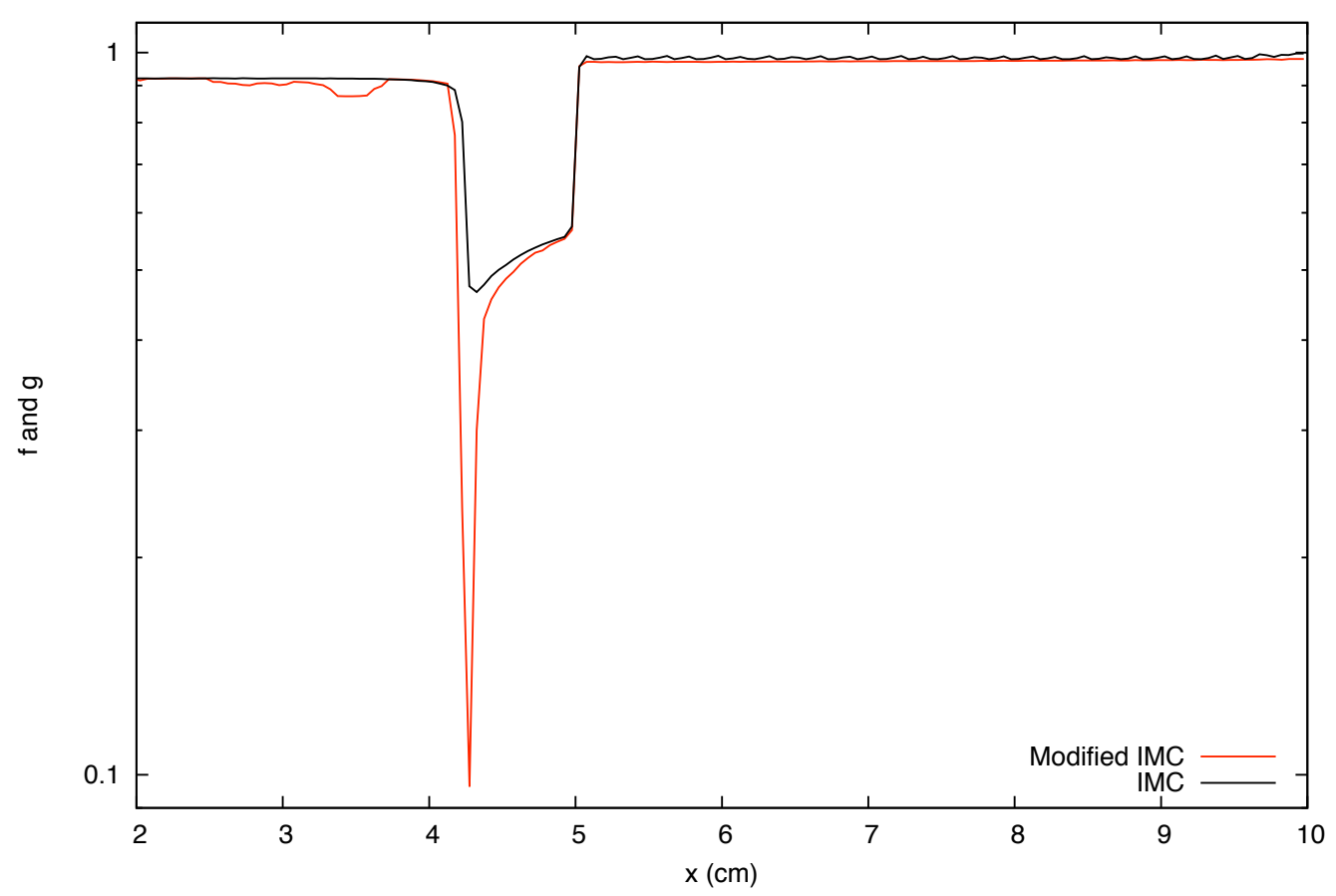

Figure 7: Values of $f$ and $g$ vs space for the Fleck and Cummings IMC and modified IMC simulations of the radiation hydrodynamics test problem. Both simulations used $\Delta t=10^{-11} \mathrm{sec}$.

(1) and (6) can then be combined into the single equation

$$
\frac{\mathrm{d} T}{\mathrm{~d} t}=\frac{c \sigma_{0} T^{-n}}{\rho c_{v}}\left[a T^{4}+\rho c_{v} T-e_{t}\right] .
$$

This equation can be cast into an integral equation for $t(T)$ :

$$
\frac{c a \sigma_{0}}{\rho c_{v}}\left(t-t_{0}\right)=\int_{T_{0}}^{T} \frac{T^{n} \mathrm{~d} T}{T^{4}+p T-q},
$$

where $T_{0} \equiv T\left(t_{0}\right), p \equiv\left(\rho c_{v}\right) / a$ and $q \equiv e_{t} / a$.

As shown in [13], the roots of the denominator of Eq. (47) can be obtained. The denominator is a quartic, and so there will be 4 roots. Since $n$ is a non-negative integer, knowing the roots of the denominator allows us to decompose the integrand by partial fractions [15]. This will let us express the integrand as a sum of a polynomial in $T$ and four terms of the form $\frac{c_{i}}{T-T_{i}}$, where $c_{i}$ is a constant and $T_{i}$ are the four roots of the denominator. The form of the polynomial and the values of the $c_{i}$ will depend on the initial temperatures and the values of the constants, such as $c_{v}$ and $\sigma_{0}$.

We will examine the specific case where $n=5$, so that the opacity has a particularly strong temperature dependence: $\sigma(t)=\sigma_{0} T^{-5}$. We will take $\sigma_{0}=10^{-3} \mathrm{~cm}^{-1} \mathrm{keV}^{-5}$. We will set $c_{v}=$ 
Table 2: Roots and coefficients for Eq. (48)

\begin{tabular}{|l|l|l|l|}
\hline root & value $(\mathrm{keV})$ & coefficient & value $\left(\mathrm{keV}^{2}\right)$ \\
\hline$T_{1}$ & $0.417340+1.535317 \mathrm{i}$ & $c_{1}$ & $-0.558980+0.487657 \mathrm{i}$ \\
$T_{2}$ & $0.417340-1.535317 \mathrm{i}$ & $c_{2}$ & $-0.558980-0.487657 \mathrm{i}$ \\
$T_{3}$ & 1.0 & $c_{3}$ & 0.130817 \\
$T_{4}$ & -1.834681 & $c_{4}$ & 0.987142 \\
\hline
\end{tabular}

$5 \times 10^{14} \mathrm{erg} /(\mathrm{g} \mathrm{keV})$. The density $\rho$ will set equal to $1.0 \mathrm{~g} / \mathrm{cm}^{-3}$. In cgs units, $c=2.9979 \times 10^{10}$ $\mathrm{cm} / \mathrm{s}$ and the radiation constant $a=1.37202 \times 10^{14} \mathrm{erg}\left(\mathrm{cm}^{3} \mathrm{keV}^{4}\right)$. The initial temperature will be $T_{0}=0.01 \mathrm{keV}$, and the initial radiation temperature will be $1.46512 \mathrm{keV}$. These values will lead to a final equilibrium temperature of $T=1.0 \mathrm{keV}$. We will take $t_{0}=0$.

With $n=5$ and the values for $c_{\nu}$, etc. given above, the integrand of Eq. (47) becomes, via partial fractions,

$$
\frac{T^{5}}{T^{4}+p T-q}=T+\frac{-p T^{2}+q T}{T^{4}+p T-q}=T+\sum_{i=1}^{4} \frac{c_{i}}{T-T_{i}},
$$

where

$$
c_{i}=\frac{p T_{i}^{2}+q T_{i}}{4 T_{i}^{3}+p}
$$

as shown in [15]. The values of $T_{i}$ and $c_{i}$ are given in Table 2. The roots $T_{1}$ and $T_{2}$ are complex conjugates, as are the constants $c_{1}$ and $c_{2}$. This holds because the integral must give a real value for the temperature. $T_{3}$ is the equilibrium temperature, $1.0 \mathrm{keV}$.

Since the first 2 roots are complex conjugates, we can combine them into a manifestly real expression:

$$
\frac{c_{1}}{T-T_{1}}+\frac{c_{1}^{*}}{T-T_{1}^{*}}=\frac{\left(c_{1}+c_{1}^{*}\right) T-\left(c_{1} T_{1}+c_{1}^{*} T_{1}^{*}\right)}{T^{2}-\left(T_{1}+T_{1}^{*}\right)+T_{1} T_{1}^{*}} .
$$

Using Eqs. (48) and (50), the integral in Eq. (47) is reduced to a sum of integrals of simple expressions for which analytic expressions can be found (see, for example [15]). These are the same forms found for the integrals in [13] with different constant coefficients. The result of the integration is

$$
\begin{aligned}
& t(T)=\frac{\rho c v}{c a \sigma_{0}} \quad\left[\frac{2 B-A C}{\sqrt{Q}} \arctan \left(\frac{2 T+C}{\sqrt{Q}}\right)\right. \\
& +\frac{A}{2} \log \left(T^{2}+C T+D\right) \\
& \left.+c_{3} \log \left(T-T_{3}\right)+c_{4} \log \left(T-T_{4}\right)\right]_{T_{0}}^{T} .
\end{aligned}
$$

The values of the constants $A, B$, etc. can be found in Table 3. This expression for $t(T)$ can be inverted by any of the standard root-finding techniques (see, e.g., [6]) to calculate values of $T$ given the time $t$. The specific values of the constants in Eq. (51) are given in Table 2. Once the value of $T(t)$ is obtained, the value of radiation temperature can be found from energy conservation.

The solution, $T(t)$ is plotted in Figure 8. This figure shows that the temperature rises very rapidly are early times, because the opacity at the initial temperature is very large. Because 
Table 3: Roots and coefficients for Eq. (51)

\begin{tabular}{|l|l|l|}
\hline coefficient & expression & value \\
\hline $\mathrm{A}$ & $c_{1}+c_{1}^{*}$ & -1.117960 \\
\hline $\mathrm{B}$ & $-\left(c_{1} T_{1}+c_{1}^{*} T_{i}^{*}\right)$ & -1.963987 \\
\hline $\mathrm{C}$ & $-\left(T_{1}+T_{1}^{*}\right)$ & -0.834681 \\
\hline $\mathrm{D}$ & $T_{1} T_{1}^{*}$ & 2.531373 \\
\hline $\mathrm{Q}$ & $4 D-C^{2}$ & 9.428800 \\
\hline
\end{tabular}

the opacity is such a strong function of temperature, it decreases as the matter is heated by the ambient radiation, so the rate of change of the temperature declines as the temperature increases.

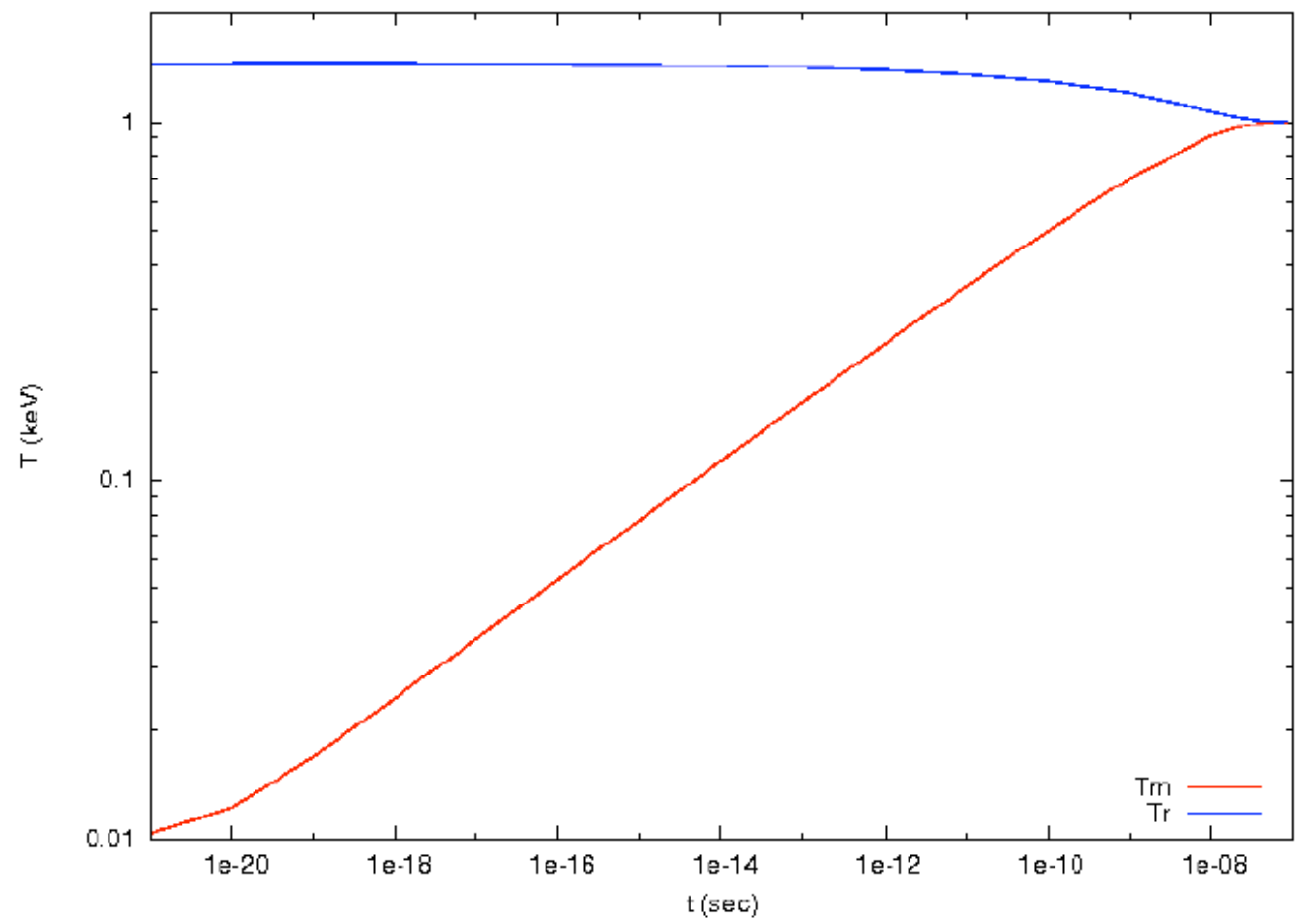

Figure 8: Semi-analytic answer for the $\sigma \sim T^{-5}$ test problem, calculated by inverting Eq. (51). Matter and radiation temperature are plotted vs. time. The temperature is in units of $\mathrm{keV}$.

\section{Acknowledgments}

This work performed under the auspices of the U.S. Department of Energy by Lawrence Livermore National Laboratory under Contract DE-AC52-07NA27344.

The author wishes to thank Ryan McClarren and George Zimmerman for interesting and useful discussions. The implementation of the Fleck and Canfield random walk algorithm in the 
Kull code was performed by Mike Lambert.

[1] J. A. Fleck, Jr. and J. D. Cummings, J. Comput. Phys. 8, 313 (1971).

[2] G. C. Pomraning, Equations of Radiation Hydrodynamics, in International Series of Monographs in Natural Philosophy, edited by D. ter Harr (Pergamon, New York, 1973), Vol. 54

[3] J. A. Fleck, Jr., in Computational Methods in the Physical Sciences (B. Alder and S. Fernbach, Eds.) Vol. 1, p.43, McGraw-Hill, New York, 1963.

[4] E. W. Larsen and B. Mercier, J. Comput. Phys. 83, 433 (1989).

[5] N. A. Gentile, International Conference on Mathematics, Computational Methods E Reactor Physics Saratoga Springs, New York, May 3-7, (2009) American Nuclear Society.

[6] Press, Teukolsky, Vetterling, and Flannery, Numerical Recipes, Third Edition (Cambridge University Press, New York, 2007).

[7] N. A. Gentile, Trans. Am. Nuc. Soc. 97, 544 (2007)

[8] S. W. Mosher and J. D. Densmore, ANS Winter Meeting and Technology Expo 93, 520 (2005).

[9] J. R. Cheatham, Ph. D. Thesis http://deepblue.lib.umich.edu/bitstream/2027.42/75852/1/cjesse_1.pdf (2010)

[10] R. G. McClarren and T. J. Urbatsch, J. Comput. Phys. 228, 5669 (2009).

[11] A. B. Wollaber, Ph. D. Thesis http://deepblue.lib.umich.edu/bitstream/2027.42/60735/1/wollaber_1.pdf (2007)

[12] N. A. Gentile, N. Keen, and J. Rathkopf, The KULL IMC Package, Tech. Rep. UCRL-JC-132743, Lawrence Livermore National Laboratory, Livermore CA (1998)

[13] S. W. Mosher, Trans. Am. Nuc. Soc. 95, 744 (2006).

[14] J. A. Fleck, Jr. and E. H. Canfield, J. Comput. Phys. 54, 508 (1984).

[15] I. S. Gradshteyn and I. M. Ryzhik, Table of Integrals, Series, and Products, Academic Press, New York, N. Y., (1980). 